\title{
DETECCIÓN DE CONDICIONES DE ENGELAMIENTO EN EL INTERIOR DE LAS MASAS NUBOSAS: APLICACIÓN A LA MEJORA DE LA SEGURIDAD AÉREA
}

J. L. Sánchez ${ }^{\text {, F. Valero² }}$, E. García-Ortega ${ }^{1}$, S. Fernández-González ${ }^{3}$, M. L. Martin", A. Merino ${ }^{1}$, J. Diaz ${ }^{1}$, L. Sánchez ${ }^{1}$, J. L. Marcos ${ }^{1}$. L. López ${ }^{1}$

(1) Grupo de Física de la Atmósfera, IMA, U. de León jl.sanchez@unileon.es (2) Dpto. de Física de la Tierra y Astrofísica, U. Complutense de Madrid valero@ucm.es

(3) Agencia Estatal de Meteorología (AEMET), Madrid, sfernandezg@aemet.es

(4) Dpto. de Matemática Aplicada. U. de Valladolid mlmartin@eii.uva.es

\section{INTRODUCCIÓN}

La causa de la formación de hielo en los aviones hay que buscarla en la presencia de agua líquida sobrenfriada (SLW) en ciertas regiones de las nubes. Al travesarlas un avión y al mero impacto con esas gotas, se puede llegar a producir su rápida congelación, dando lugar al proceso denominado engelamiento. Hay dos mecanismos que generan SLW. El primero es cuando tenemos hidrometeoros que se descongelan al atravesar una región en la que la temperatura se encuentra por encima de $0^{\circ} \mathrm{C}$ y, posteriormente, en su caída vuelven a entrar en zonas donde la temperatura vuelve a estar algo por debajo de $0{ }^{\circ} \mathrm{C}$, con lo que no llegan a congelar y se forma SLW. Este mecanismo se encuentra favorecido por la formación de inversiones térmicas cerca de la superficie. El segundo mecanismo consiste en la formación de gotas líquidas en regiones nubosas en las que la temperatura permanece por debajo de $0{ }^{\circ} \mathrm{C}$ y tienen dificultades para congelar (Fernández-González et al., 2015). Las gotas pueden crecer mediante los procesos de colisión seguido de coalescencia, lo que puede dar lugar a la formación de grandes gotas sobrenfriadas (SLD), con un tamaño superior a $50 \mu \mathrm{m}$ de diámetro. Las SLD son muy peligrosas para la aviación ya que pueden provocar la acumulación de hielo en lugares del fuselaje no protegidos suficientemente mediante sistemas anti-engelamiento. Por tanto, el engelamiento es producto de la combinación de SLW y de SLD. La región de la troposfera con mayor riesgo de engelamiento provocado por la presencia de SLD suele encontrarse en el rango de temperaturas comprendido entre -2 y $-20^{\circ} \mathrm{C}$ (Ledesma y Baleriola, 2003).

La carga de hielo que producen las SLD en los bordes de ataque del avión conlleva la pérdida de sustentación y cuando es extrema, se producen incidentes cuando no accidentes aéreos. Para aumentar la seguridad aeronáutica, es conveniente hacer uso de modelos numéricos de predicción meteorológica que establezcan las zonas en las que puede aparecer SLW (Fernández-González et al., 2014). Como cualquier modelo debe validarse, y para ello se necesitan datos experimentales de medidas "in situ", lo cual no es sencillo, pues hay que entrar en el interior de las nubes, efectuar medidas de microfísica de nubes y además hacerlo en condiciones razonables de seguridad. Hay que tener en cuenta que las simulaciones numéricas tienden a sobreestimar la presencia y cantidad de contenido de agua en fase sólida, mientras que subestiman la existencia y concentración de SLW (Vaillancourt et al., 2002). 
Realizar vuelos científicos en estas condiciones resulta arriesgado por lo que hay que tomar una serie de precauciones que van desde establecer procedimientos de vuelo hasta colocar instrumentación meteorológica que amplíe la observación que se realiza desde las redes habituales. Si se hace adecuadamente, también se pueden obtener medidas indirectas que señalen la presencia de SLW.

En este trabajo se describen algunas de las acciones que se establecieron para garantizar tanto la operación de vuelo como la toma de datos de microfísica de nubes en el transcurso de diferentes campañas realizadas entre 2011 y 2017.

\section{DISEÑO EXPERIMENTAL}

El Grupo de Física de la Atmósfera (GFA) de la Universidad de León en colaboración en ocasiones con la Universidad Complutense de Madrid, viene realizando desde hace años numerosos trabajos de investigación acerca de las condiciones y causas en las que se produce el engelamiento. Actualmente hay en curso una serie de actividades en el marco del proyecto SAFEFLIGHT financiado por el Plan Nacional de Investigación. Dentro de este proyecto se analizan los datos recogidos en las campañas experimentales de otros dos Proyectos también financiados por el Plan Nacional: TECOAGUA (desarrollada entre los años 2011 y 2013, y centrada en estudiar las condiciones en la sierra de Guadarrama) y METEORISK (en la que se realizaron vuelos entre los años 2016 y 2017 en el macizo Galaico-Leonés y la parte más occidental de la cordillera Cantábrica). Estas campañas se han desarrollado en colaboración con el Instituto Nacional de Técnica Aeroespacial (INTA).

Todos los vuelos científicos de toma de datos se realizaron en época invernal y en condiciones meteorológicas favorables para la formación de regiones con SLW. El objetivo principal de todos los vuelos fue el de detectar y caracterizar las zonas en las que aparecía SLW y, cuando era posible, detectar las SLD.

Se han empleado dos tipos de instrumentos: los situados en la plataforma aérea C212, que pertenece a las denominadas Infraestructuras Científicas y Técnicas Singulares (ICTS) y los que situados en el suelo daban información en modo continuo. Los primeros detectan directamente las condiciones que potencialmente pueden provocar engelamiento y las segundas lo hacen de forma indirecta.

\subsection{Medidas desde la plataforma C212:}

El instrumento principal es una CAPS (Cloud, Aerosol and Precipitation Spectrometer), mostrado en la Figura 1. Como se puede ver se encuentra instalado en el intradós del ala izquierda. Se compone de los siguientes instrumentos:

- Cloud Imaging Probe Grayscale (CIP-GS), capaz de medir el tamaño de los hidrometeoros en un rango entre 7,5 $\mu \mathrm{m}$ y $930 \mu \mathrm{m}$. El sensor de este instrumento, compuesto por emisores y receptores láser, presenta así una banda de medición de 62 canales con una resolución de $15 \mu \mathrm{m}$. Cuando el nivel de luz disminuye en un $50 \%$, el estado del diodo pasa a estar activado, detectándose que existe un hidrometeoro entre el emisor láser y el receptor y clasificándolo según su tamaño. La representación de los hidrometeoros detectados en imagen 2D permite identificar asimismo el estado físico de los mismos 
a partir de sus propiedades ópticas. La sonda CIP es utilizada para calcular el número de hidrometeoros que atraviesan cada canal, así como el diámetro medio volumétrico (MVD) y el diámetro efectivo (ED).

- Cloud and Aerosol Spectrometer (CAS) es una sonda que realiza mediciones tanto de los distintos tamaños de aerosoles como de los diferentes hidrometeoros presentes en las nubes, para un tamaño comprendido entre 0,5-50 $\mu \mathrm{m}$, complementando así los datos de la CIP para tamaños menores.

- Liquid Water Content Detector (Hotwire LWC), que permite estimar con precisión el contenido de agua líquida (LWC) presente en la atmósfera para el rango $0,01-3 \mathrm{~g} / \mathrm{m}^{3}$. El contenido de agua líquida es calculado mediante la potencia eléctrica que hay que suministrar a una resistencia térmica para mantener una temperatura constante (alrededor de unos $\left.125^{\circ} \mathrm{C}\right)$.

- La sonda CAPS dispone asimismo de un GPS para conocer en todo momento la altitud, latitud y longitud (y de esa manera determinar correctamente los tramos de los vuelos con diferentes condiciones), así como sensores de temperatura y humedad, y un tubo Pitot para poder medir la velocidad del viento. Con todo ello se puede conocer el volumen de aire muestreado, los hidrometeoros detectados y su posición en cada momento.

A partir de los datos recogidos por los instrumentos que forman parte del equipo CAPS se han podido caracterizar las condiciones nubosas que la aeronave se ha encontrado durante los vuelos experimentales. Entre los productos obtenidos más importantes destacan las frecuencias del LWC (Figura 2). Debido a que son medidas realizadas en nubes invernales el valor del LWC suele ser bajo, por lo que se ha representado en escala logarítmica. Como se puede observar, la frecuencia va disminuyendo según aumenta la concentración de LWC, detectándose en el 95 \% de las mediciones realizadas concentraciones menores de $1 \mathrm{~g} / \mathrm{m}^{3}$.

\subsection{Instrumentación situada en el suelo:}

Esta instrumentación se situó con el objetivo de detectar indirectamente la presencia de SLW y las condiciones meteorológicas en las que se formaban. Además, en este apartado también describimos otros tipos de instrumentos que complementaban el registro

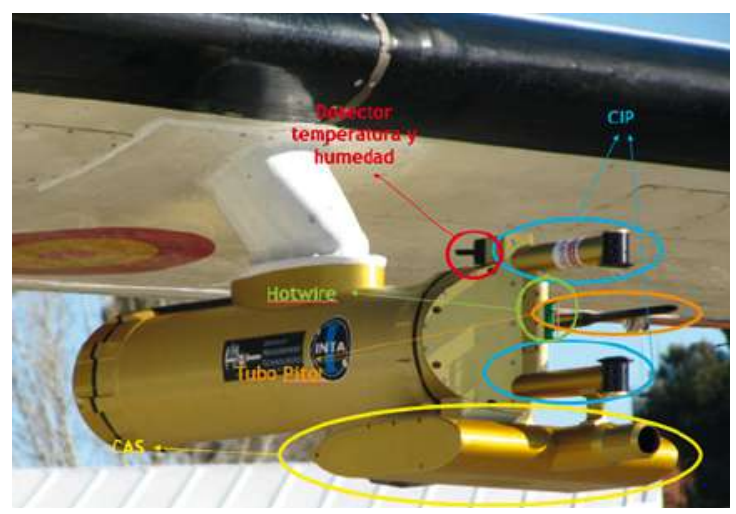
de precipitación o que ayudaban a mejorar la información a la tripulación y, por tanto, la seguridad del avión.

La utilización de multiplataformas con tecnologías diferentes que permiten establecer las características de las masas nubosas fue uno de

Fig. 1.- CAPS instalada en el intradós del C212 
los requisitos que se establecieron desde el principio para la mejor planificación de los vuelos y para mejorar la seguridad de las operaciones aéreas. El hecho de que se empleen mejora la información y permite obtener detalles que de otra forma resulta más difícil. En las figuras 3 y 4 se muestra la mayor parte de la instrumentación utilizada.

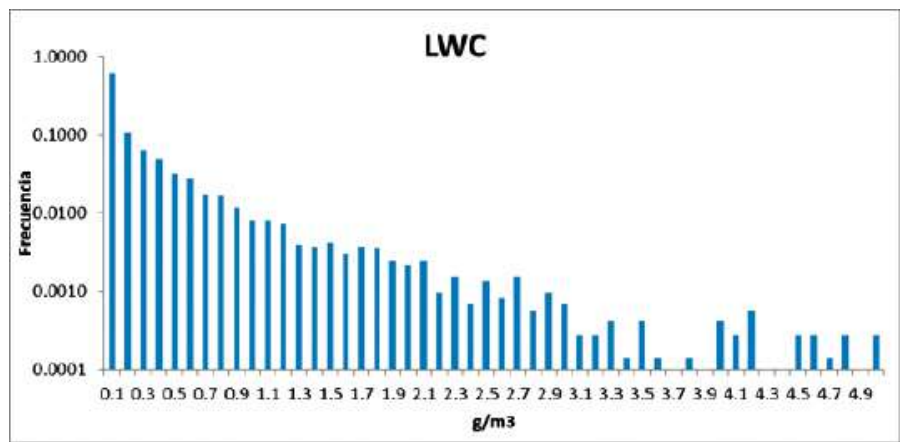

Fig. 2.- Frecuencia de aparición del contenido de agua líquida (LWC) encontrado en los vuelos científicos en los que se produjo engelamiento de tipo "hielo transparente" en los perfiles de las alas aún con los sistemas antihielo activados. En abscisas se presentan los intervalos del LWC $\left(\mathrm{g} / \mathrm{m}^{3}\right)$.

Fig. 3.- Instrumentación instalada en el suelo para el desarrollo de las Campañas de Vuelos y de los proyectos TECOAGUA, METEORISK y SAFELIGHT. Se trata (de izquierda a derecha) del VPF 730, Micro Radar, Radiómetro de Microondas y Ceilómetro.
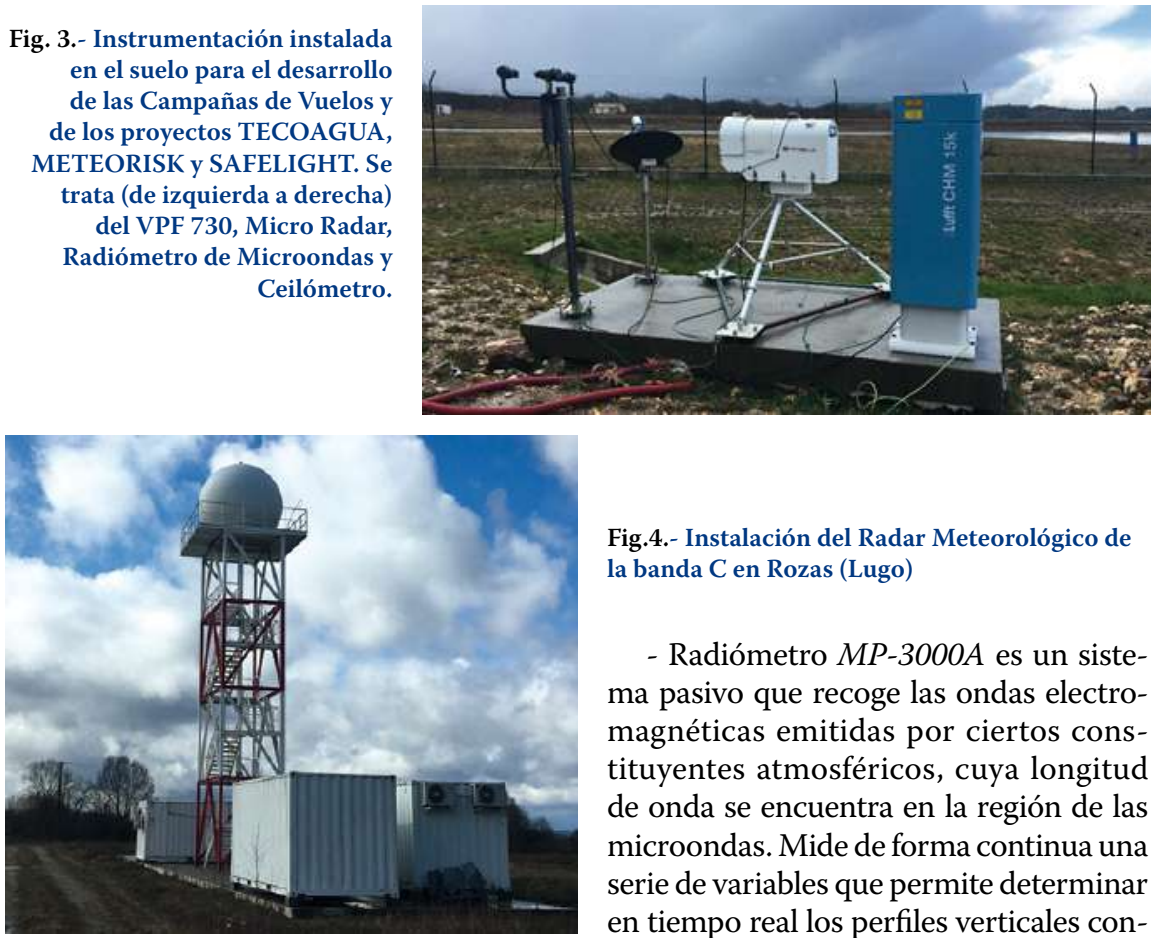

Fig.4.- Instalación del Radar Meteorológico de la banda C en Rozas (Lugo)

- Radiómetro MP-3000A es un sistema pasivo que recoge las ondas electromagnéticas emitidas por ciertos constituyentes atmosféricos, cuya longitud de onda se encuentra en la región de las microondas. Mide de forma continua una serie de variables que permite determinar en tiempo real los perfiles verticales con- 
tinuos de temperatura, humedad, vapor de agua y agua líquida. En la figura 5 se muestra un ejemplo con los registros tomados en uno de los días en los que se detectaron regiones con SLW. En este caso se puede ver que se trata de regiones con SLW ya que la temperatura se sitúa por debajo de $0{ }^{\circ} \mathrm{C}$. Esta forma de aparición en forma de "bolsas" discontinuas es muy típica del LWC. Además, van apareciendo y desapareciendo en el transcurso del tiempo. Este es un fenómeno muy característico de las regiones que potencialmente pueden producir engelamiento en los aviones. El tratamiento de la información permite minimizar los errores de la medida (Sanchez al., 2013)

- El Micro Rain Radar que trabaja en la frecuencia de $24 \mathrm{GHz}$. Obtiene un perfil vertical de la atmósfera en 31 niveles, con una resolución en altura regulable entre 10 y 1000 metros, y una resolución en el tiempo también regulable, entre 10 y 3600 segundos. Este radar permite determinar en la vertical las intensidades de la precipitación, el nivel de fusión y de forma más aproximada, el perfil del LWC.

- El disdrómetro óptico VPF-730 es un instrumento muy utilizado en aeropuertos y carreteras ya que detecta diferentes tipos de precipitación como pueden ser lluvia o nieve. Además de determinar la intensidad y cantidad de precipitación, puede medir otras variables como visibilidad, precipitación líquida y sólida (lluvia, nieve, granizo, etc.). Con este instrumento se puede seguir en modo continuo el tipo de precipitación en el suelo.

- Ceilómetro: basado en tecnología lídar, su uso está orientado a conocer las características de la capa límite, los perfiles verticales de visibilidad (aerosoles) y especialmente, a detectar capas de nubes, alturas de las bases y techos de nubes. El que se ha utilizado en este caso es el CHM 15k "NIMBUS". En las campañas de vuelos contribuyó a mejorar la información de la base y el techo de nubes.

- Radar meteorológico: de la banda C (5450 a $5825 \mathrm{MHz}$ ) con una potencia pico de $250 \mathrm{~kW}$ con un PRF de 250 pps. Se trata de un Interprise WR100-2/77 al que interiormente se ha ido actualizando periódicamente y que proporciona imágenes digitalizadas. Se viene utilizando desde hace años en las campañas de medidas del GFA (López y Sánchez, 2009; Sánchez et al., 2013). Tiene un sistema de adquisición de datos configurable con un alcance de $140 \mathrm{~km}$ aproximadamente y habitualmente se opera de forma que se

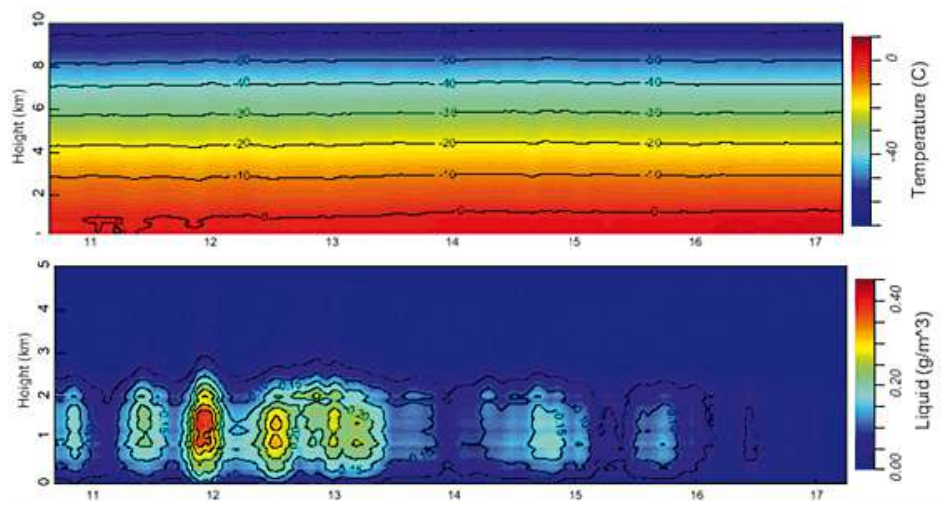

Fig.5.- En la parte superior se puede ver la evolución de las isotermas. En la inferior se puede apreciar la aparición de SLW formando "bolsas" e irregularmente repartidas. 
obtienen imágenes cada 4 minutos aproximadamente. Su uso ha tenido la finalidad de proporcionar información de conjunto a los pilotos de la plataforma C212 y mejorar la seguridad de los vuelos.

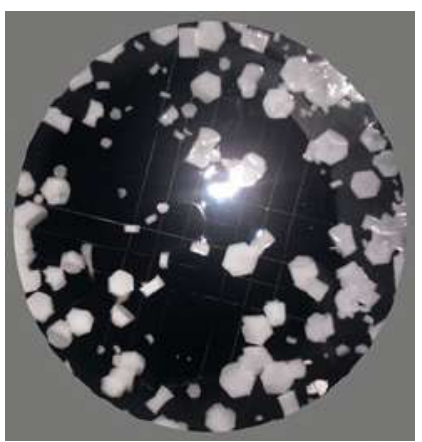

- Cámara estática de medida de núcleos de congelación (IN) o cámara de nubes: se trata de un dispositivo que es capaz de producir la saturación en un ambiente de temperatura controlado y activar la formación de núcleos de congelación (Castro et al., 1998). Resulta útil para complementar la información de las condiciones ambientales en superficie. En la figura 6 se puede ver una muestra de los IN activados.

Fig. 6.- Sobre el fondo de la cámara de nubes se observan los cristales de hielo formados a partir de la activación de los IN.

\section{CASO DE ESTUDIO: VUELO DEL 23 DE MARZO DE 2017}

Hemos seleccionado el vuelo realizado el 23 de marzo de 2017 con origen en la base aérea de Torrejón de Ardoz (Madrid) y destino el aeropuerto de León, siguiendo la trayectoria que se puede observar en la Figura 7. El vuelo se realizó entre las 10:30 y las 12:00 (hora local) a una altitud máxima de 3700 metros; la temperatura durante el

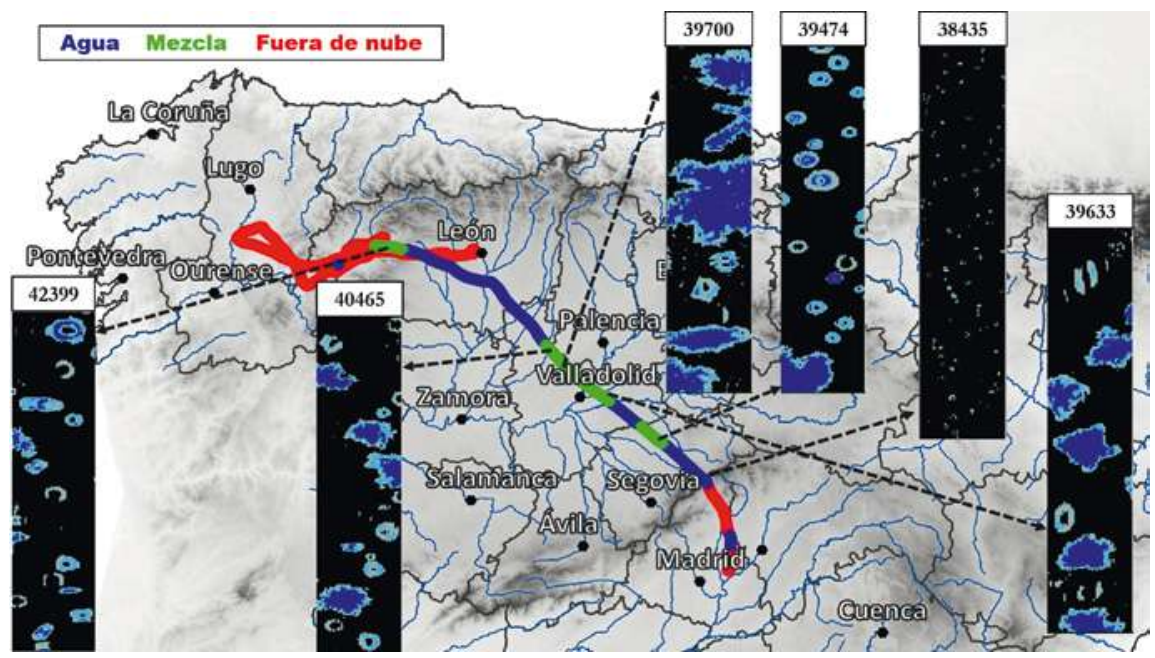

Fig.7.- Trayectoria del vuelo del 23 de marzo de 2017 entre Madrid y León e imágenes extraídas de la CIP durante el vuelo, en relación a los diferentes tramos en que se dividió el vuelo a partir de las condiciones encontradas. 
trayecto osciló entre los -14 y los $-8^{\circ} \mathrm{C}$. El piloto notificó detección de engelamiento durante el vuelo en diversos puntos, en todos los casos coincidentes con SLD.

Se realizó posteriormente un análisis de los datos e imágenes recogidas por la CIP. Se representaron en color azul aquellos tramos en los que se detectó agua líquida. Por ejemplo, en la imagen 38435 se observan cloud droplets (gotas de agua líquida de diámetro inferior a $50 \mu \mathrm{m}$ ) a una temperatura de $-12,3{ }^{\circ} \mathrm{C}$ y a 1260 metros de altitud. Por su parte, en color verde se representaron los tramos en los que se observó una mezcla de agua y hielo. En la imagen 39474 se observan SLD a $-7,4^{\circ} \mathrm{C}$. En la imagen 40465 , se puede observar como coexisten gotas en fase líquida y en fase sólida; la presión de vapor del hielo es menor que la del agua líquida, por lo que las moléculas de agua se depositan sobre la superficie de los hidrometeoros en fase sólida, produciéndose además la evaporación de las gotitas de agua más pequeñas; este proceso es conocido como Bergeron-Findeisen (Fernández-González et al., 2014). Por último, a $-14,6^{\circ} \mathrm{C}$ se puede observar un ejemplo de colisión-coalescencia (imagen 42399). En la imagen 39700 se puede observar la formación de graupel a $-9,1^{\circ} \mathrm{C}$. Finalmente, los tramos en color rojo indican la ausencia de imágenes de la CIP, lo que significa que el avión volaba fuera de nube.

Hay que destacar la complejidad de estos vuelos, ya que el avión dentro de la nube puede sufrir engelamiento, por lo que debe entrar y salir constantemente para no acumular demasiado hielo; esto se traduce en continuas subidas y bajadas de la aeronave. Por ello, se deben seleccionar tramos del trayecto de cada vuelo en los que la altitud sea más o menos constante $( \pm 200 \mathrm{~m})$ para poder analizar correctamente la información de los sensores.

Los datos acerca de las condiciones atmosféricas encontradas durante los vuelos resultan de suma importancia a la hora de caracterizar las condiciones favorables al engelamiento y, por tanto, para la precisión y la mejora en cuanto a la seguridad del transporte aéreo. Con este fin último, los datos recogidos en los vuelos pueden ser empleados en el futuro como medidas "in situ" fundamentales para la construcción y validación de modelos numéricos de predicción meteorológica que determinen las zonas donde pueda aparecer SLW. De esta manera se espera poder pronosticar en tiempo y lugar las condiciones de engelamiento, permitiendo la construcción de planes y rutas de vuelo más seguros que eviten en lo posible las áreas y capas de la atmósfera con condiciones más peligrosas.

\section{DISCUSIÓN Y CONCLUSIONES}

La detección de áreas con riesgo de engelamiento puede efectuarse de forma directa e indirecta. En los dos casos si se pretende ir más allá del simple análisis de los entornos meteorológicos favorables para la formación de engelamiento en aviones, debe de actuarse con mucho cuidado y una buena planificación. La caracterización de las regiones con SLW puede hacerse de forma directa mediante el uso de plataformas aéreas que deben contar con planes específicos de seguridad en el vuelo. Además, deben disponer de instrumentación de microfísica de nubes y protocolos de vuelo específicos.

La utilización de multiplataformas con instrumentación en el suelo facilitan la información a la tripulación durante los vuelos, mejorando la seguridad de los mismos. 
Al comparar la información suministrada por la instrumentación del avión con la de la situada en el suelo, se mejora la interpretación de los datos. Los resultados obtenidos muestran que contenidos de agua líquida cercanos a 0,1 g/m3, si se detectan SLD, hacen que la probabilidad de que se produzca carga de hielo en las partes más expuestas del fuselaje de un avión sea muy elevada. La aparición de estas regiones suele ser formando bolsas y más probables en las cercanías de las bases de las nubes, en donde se produce una acumulación de contenido SLW.

La clasificación de los tramos en los que se detectan tipos de hidrometeoros, contribuye a mejorar la calibración y validación de los modelos mesoescalares que son las herramientas fundamentales que mejorarán la seguridad de los vuelos, especialmente en las operaciones de aproximación y despegue.

Agradecimientos: Este trabajo se ha realizado en el marco del Proyecto SAFEFLIGHT financiado por el MINECO (CGL2016-78702). Los autores del mismo quieren agradecer al INTA y a las tripulaciones del CLAEX las facilidades que nos han dado a lo largo de los años. Una parte de estos Proyectos se realizaron en el CIAR en Rozas (Lugo). También queremos destacar el agradecimiento por la ayuda prestada por R. Weigand dando soporte informático al grupo investigador y por Neves Seone por el enorme esfuerzo que ha realizado desde el INTA para hacer posible los vuelos y el manejo de los datos de la CASP.

\section{REFERENCIAS}

- Castro A., Marcos, J.L., Dessens, J., Sánchez, J.L., Fraile, R. 1998: Concentration of Ice Nuclei in Continental and Maritime Air Mases in León (Spain). Atmospheric Research. 47-48, pp. 155 - 167.

- Fernández-González, S., Sánchez, J.L., Gascón, E., López, L., García-Ortega, E., Merino, A. 2014: Weather features associated with aircraft icing conditions: A case study. The Scientific World Journal Volume 2014.

- Fernández-González, S., Valero, F., Sánchez, J.L., Gascón, E., López, L., García-Ortega, E., Merino, A. 2015: Analysis of a seeder-feeder and freezing drizzle event. Journal of Geophysical Research 120, 3984-3999.

- Ledesma, M., and Baleriola, G., 2003: Meteorología Aplicada a la Aviación. S.A. Ediciones Paraninfo. 544 pp.

- Lopez, L., and Sánchez, J.L. 2009: Discriminant methods for radar detection of hail. Atmospheric Research. 93, pp.358-368

- Sanchez, J.L. et al. 2009: Assessing sounding-derived parameters as storm predictors in different latitudes. Atmospheric Research. 93, pp.446-456.

- Sanchez, J.L. et al. 2013 : Nowcasting of kinetic energy of hail precipitation using radar. Atmospheric Research. 123, pp.48-60. 